\title{
Predicting the Permanent Safe Donor Area for Hair Transplantation in Koreans with Male Pattern Baldness according to the Position of the Parietal Whorl
}

\author{
Jae Hyun Park ${ }^{1}$, Young Cheon $\mathrm{Na}^{2}$, Jae Seong $\mathrm{Moh}^{1}$, Seung Yong Lee ${ }^{1}$, Seung Hyun You ${ }^{1}$ \\ ${ }^{1}$ Dana Plastic Surgery Clinic, Seoul; ${ }^{2}$ Department of Reconstructive and Plastic Surgery, Wonkwang University Hospital, Wonkwang \\ University School of Medicine, Iksan, Korea
}

\begin{abstract}
Background The most crucial factor in hair transplantation for male pattern baldness (MPB) patients is the efficient utilization of the donor-recipient ratio. However, there is no known factor that scientifically predicts the rate of progression of alopecia or indicates a permanently safe donor area.

Methods The study considered 1,008 Korean adult males with MPB; of these, it excluded 56 males with an absence of parietal whorls (PWs). The authors investigated the distance from the vertical bimeatal line (VM) to the PW, from the PW to the upper border of the helical rim $(\mathrm{HR})$, and the distance from the PW to the occipital fringe (OF) in 952 subjects with a PW. Furthermore, we examined the distance from the PW to the OF considering the duration of alopecia and age in 322 subjects with vertex alopecia.

Results The distance between the VM and PW varied from 1.5 to $11 \mathrm{~cm}$, with an average distance of $6.25 \mathrm{~cm}$. The PW-HR distance ranged from 3.4 to $17.5 \mathrm{~cm}$, and the average distance was $7.79 \mathrm{~cm}$. The PW-OF distance ranged from 0.5 to $5.5 \mathrm{~cm}$, and the average distance was $2.37 \mathrm{~cm}$.

Conclusions For the PW, very large variations existed in the vertical direction. The position of the PW could predict the progression range of the total alopecia of the vertex. Alopecia mostly progresses within $6 \mathrm{~cm}$ of the PW toward the occipital side.
\end{abstract}

Keywords Alopecia / Hair follicle / Transplant donor sites
Correspondence: Jae Hyun Park Dana Plastic Surgery Clinic, $11 \mathrm{~F}$ Human Tower, 605 Gangnam-daero, Seocho-gu, Seoul 137-903, Korea Tel: +82-2-512-0922

Fax: +82-2-512-0942

E-mail: jay8384@naver.com

\section{INTRODUCTION}

The most important factors in hair transplantation of male pattern baldness (MPB) are precise assessment and management of the donor area, and the designing and planning of the recipient area by taking long-term progress into consideration.
Hair transplantation has drawn more attention among patients these days because of the recent advances in surgical techniques. Consequently, hair transplant surgeons have been trying to transplant the maximum-possible number of hair grafts in a single session to cover more of the recipient area.

Recently, a large number of grafts have been obtained and

Copyright $(\odot 2014$ The Korean Society of Plastic and Reconstructive Surgeons

This is an Open Access article distributed under the terms of the Creative Commons Attribution Non-Commercial License (http://creativecommons.org/

licenses/by-nc/3.0/) which permits unrestricted non-commercial use, distribution, and reproduction in any medium, provided the original work is properly cited.

www.e-aps.org

No potential conflict of interest relevant to this article was reported. 
transplanted using follicular unit extraction (FUE). In these cases, many surgeons have frequently been in dispute about the appropriate standards for defining a safe donor area.

A safe donor area is an area that is expected to be permanently covered with hair for a lifetime and therefore, provide permanent coverage when transplanted to the recipient area.

However, scientific determination of the prognosis of the extent of MPB and a permanent donor area is impossible.

\section{METHODS}

Out of the 1,008 subjects with MPB who had no history of using finasteride or minoxidil, 43 cases were excluded as they had experienced procedures that could have affected the position of the parietal whorl (PW) position such as loss of the PW, a his- tory of excision of a soft tissue tumor on the side of the occiput, or a history of hair transplantation by the strip method. Thirteen subjects had a double whorl. The double whorl was excluded for accurate measurement. Thus, as 56 subjects were excluded from the original set of 1,008 subjects, the PW's position was measured in a total of 952 subjects.

The horizontal plane connecting the upper borders of both helical rims (HR) and the vertical bimeatal line (VM), which connects the bilateral external acoustic meatus by a coronal plane, were the basis of the measurement points (Fig. 1).

First, we measured the distance from the VM to the center of the PW, as well as the distance from the horizontal plane, which connects the upper border of the HR, to the center of the whorl. The sum of these two measurements was VM-HR. This parameter was used for identifying the vertical positional variation of

Fig. 1. Landmarks of measurement for parietal whorl position

(A) Illustration. (B, C) Clinical photograph. VM, vertical bimeatal line; PW, parietal whorl; OF, occipital fringe; HR, horizontal plane, which connects the upper border of the helical rim.
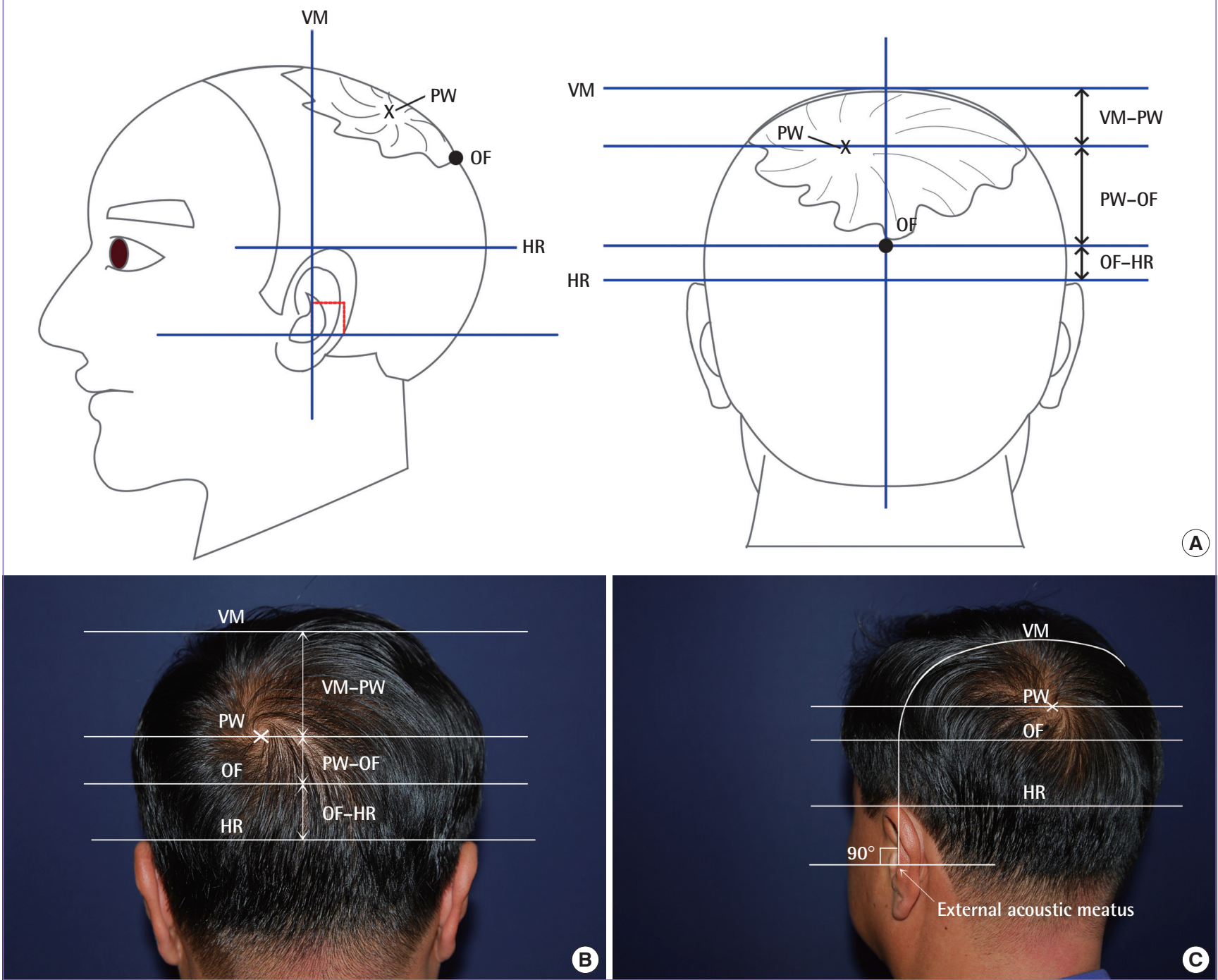
the PW.

Secondly, we measured the distance from the center of the PW to the occipital fringe (OF) on the posterior side in 322 subjects with vertex alopecia. The existence of the $\mathrm{OF}$ was assumed at a density of 64 hairs $/ \mathrm{cm}^{2}[1]$.

\section{RESULTS}

The measurement results are as shown in the following tables: the PW was located at a distance of $6.25 \mathrm{~cm}$ from the VM on average, but there was some variation, with values ranging from 1.5 to $11 \mathrm{~cm}$ (Table 1). The mean distance was $6.25 \mathrm{~cm}$. It is interesting to note that there was substantial variation in the vertical position of the PW (Table 2).

The distance from the PW to the horizontal plane, which connects the upper border of the $\mathrm{HR}$, was $7.79 \mathrm{~cm}$ on average, with a distribution ranging from 3.1 to $12 \mathrm{~cm}$. Thus, it was inferred that the VM-PW distance and the PW-HR distance were inversely proportional to each other.

The VM-HR distance ranged from 10 to $17.5 \mathrm{~cm}$ with a mean value of 14.04. This distance was associated with the size of the head. The VM-HR distance had a standard deviation of 1.07, and this was comparatively consistent. A total of 800 subjects out of 952 had a VM-HR distance of 13 to $16 \mathrm{~cm}$ (84\%); these subjects were considered to exhibit a comparatively consistent pattern.

The distance from the PW to the OF was $2.37 \mathrm{~cm}$ on average. Its distribution ranged from 0.5 to $5.5 \mathrm{~cm}$ (in a total of 322 cases).

Further, we classified the subjects into groups according to their ages: $20-29,30-39,40-49,50-59,60-69,70-79$, and $\geq 80$ years old. Subjects older than 65 years old were classified into subgroups of 65-69, 70-74, 75-79, and $\geq 80$ years old.

There was no significant difference in the PW-OF distance by age group identified from the analysis (Tables 3,4 ).

The mean distances were compared on the basis of the duration of alopecia.

The distance was investigated in five-year units, depending on the duration of the alopecia $(1-5,6-10,11-15,16-20,21-25$, $26-30$, and $\geq 31$ years) (Table 5).

\section{Table 2. Measured values from each landmark}

\begin{tabular}{|lcccc|}
\hline $\begin{array}{l}\text { Measured values/ } \\
\text { Landmark }\end{array}$ & VM-PW & PW-HR & VM-HR & PW-OF \\
\hline Number & 952 & 952 & 952 & 322 \\
$\begin{array}{l}\text { Mean } \pm \text { standard } \\
\text { deviation (cm) }\end{array}$ & $6.25 \pm 1.7$ & $7.79 \pm 1.56$ & $14.04 \pm 1.07$ & $2.37 \pm 1.06$ \\
Minimum (cm) & 1.5 & 3.4 & 10.0 & 0.5 \\
Maximum (cm) & 11.0 & 12.0 & 17.5 & 5.5 \\
\hline
\end{tabular}

VM, vertical bimeatal line; PW, parietal whorl; $H R$, upper border of helical rim; $\mathrm{OF}$, occipital fringe.

\section{Table 1. Variation in the parietal whorl position}

\begin{tabular}{|c|c|c|c|c|c|c|c|c|c|c|c|c|}
\hline $\begin{array}{l}\text { VM-PW } \\
\text { distance }(\mathrm{cm})\end{array}$ & $\begin{array}{c}>1 \text { and } \\
\leq 2\end{array}$ & $\begin{array}{c}>2 \text { and } \\
\leq 3\end{array}$ & $\begin{array}{c}>3 \text { and } \\
\leq 4\end{array}$ & $\begin{array}{c}>4 \text { and } \\
\leq 5\end{array}$ & $\begin{array}{c}>5 \text { and } \\
\leq 6\end{array}$ & $\begin{array}{c}>6 \text { and } \\
\leq 7\end{array}$ & $\begin{array}{c}>7 \text { and } \\
\leq 8\end{array}$ & $\begin{array}{c}>8 \text { and } \\
\leq 9\end{array}$ & $\begin{array}{c}>9 \text { and } \\
\leq 10\end{array}$ & $\begin{array}{c}>10 \text { and } \\
\leq 11\end{array}$ & $11 \leq$ & Total \\
\hline Number & 2 & 18 & 60 & 108 & 182 & 230 & 178 & 118 & 42 & 8 & 6 & 952 \\
\hline
\end{tabular}

Table 3. Distances between the parietal whorl and occipital fringe according to the patient's age

\begin{tabular}{|c|c|c|c|c|c|c|c|c|}
\hline Age (yr) & $20-29$ & $30-39$ & $40-49$ & $50-59$ & $60-69$ & $70-79$ & $\geq 80$ & Total \\
\hline Number & 4 & 24 & 26 & 34 & 82 & 120 & 32 & 322 \\
\hline $\begin{array}{l}\text { Mean } \pm \text { standard } \\
\text { deviation }(\mathrm{cm})\end{array}$ & $2.92 \pm 0.09$ & $2.31 \pm 1.11$ & $2.23 \pm 1.07$ & $2.26 \pm 1.19$ & $2.40 \pm 1.05$ & $2.39 \pm 1.04$ & $2.46 \pm 1.11$ & $2.37 \pm 1.06$ \\
\hline Minimum (cm) & 2.8 & 0.7 & 1.0 & 0.5 & 0.5 & 0.5 & 0.5 & 0.5 \\
\hline Maximum (cm) & 3.0 & 4.0 & 5.0 & 5.0 & 4.5 & 5.5 & 4.4 & 5.5 \\
\hline
\end{tabular}

\section{Table 4. Distances between the parietal whorl and occipital fringe according to the patient's age (older than 65 years)}

\begin{tabular}{|lccccc|}
\hline Patient's age $(\mathrm{yr})$ & $\mathbf{6 5 - 6 9}$ & $\mathbf{7 0 - 7 4}$ & $\mathbf{7 5 - 7 9}$ & $\mathbf{2 0}$ & Total \\
\hline Number & 56 & 56 & 64 & 32 & 208 \\
Mean \pm standard deviation $(\mathrm{cm})$ & $2.63 \pm 1.08$ & $2.21 \pm 0.93$ & $2.54 \pm 1.11$ & $2.46 \pm 1.11$ & 0.5 \\
Minimum (cm) & 0.5 & 0.5 & 0.5 & 4.4 & 0.5 \\
Maximum (cm) & 4.5 & 4.6 & 5.5 & & 5.5 \\
\hline PW, parietal whorl; OF, occipital fringe. & & & & \\
\hline
\end{tabular}


Table 5. Distances between the parietal whorl and occipital fringe classified by duration of alopecia

\begin{tabular}{|c|c|c|c|c|c|c|c|c|}
\hline $\begin{array}{l}\text { Duration of } \\
\text { alopecia (yr) }\end{array}$ & $1-5$ & $6-10$ & $11-15$ & $16-20$ & $21-25$ & $26-30$ & $31 \leq$ & Total \\
\hline Number & 14 & 60 & 60 & 50 & 48 & 24 & 66 & 322 \\
\hline $\begin{array}{l}\text { Mean } \pm \text { standard } \\
\text { deviation }(\mathrm{cm})\end{array}$ & $2.19 \pm 1.05$ & $2.21 \pm 1.04$ & $2.16 \pm 1.07$ & $2.42 \pm 1.18$ & $2.34 \pm 0.88$ & $2.66 \pm 0.96$ & $2.63 \pm 1.11$ & $2.37 \pm 1.06$ \\
\hline Minimum (cm) & 1.0 & 0.5 & 0.5 & 0.5 & 1.0 & 1.5 & 1.0 & 0.5 \\
\hline Maximum (cm) & 4.0 & 4.5 & 4.4 & 5.0 & 4.5 & 5.0 & 5.5 & 5.5 \\
\hline
\end{tabular}

Table 6. The cumulative frequency and percentage of distances from the PW to the OF

\begin{tabular}{|lcc|}
\hline $\begin{array}{l}\text { PW-OF } \\
\text { distance }(\mathbf{c m})\end{array}$ & $\begin{array}{c}\text { Accumulative } \\
\text { frequency }\end{array}$ & $\begin{array}{c}\text { Accumulative } \\
\text { percentage }(\%)\end{array}$ \\
\hline 1 & 50 & 15.5 \\
2 & 136 & 42.2 \\
3 & 252 & 78.3 \\
3.6 & 292 & 90.7 \\
4 & 302 & 93.8 \\
4.3 & 306 & 95.0 \\
5 & 320 & 99.4 \\
5.5 & 322 & 100 \\
\hline PW, parietal whorl; OF, occipital fringe. & \\
\hline
\end{tabular}

The mean distance for 1 to 5 years was $2.19 \mathrm{~cm}$; for 6 to 10 years, $2.21 \mathrm{~cm}$; for 11 to 15 years, $2.16 \mathrm{~cm}$; for 16 to 20 years, $2.42 \mathrm{~cm}$; for 21 to 25 years, $2.34 \mathrm{~cm}$; for 26 to 30 years, $2.66 \mathrm{~cm}$; and for $\geq 31$ years, $2.37 \mathrm{~cm}$. The duration of alopecia and the mean distance were proportional, but there was no statistical significance $(\mathrm{P}=0.123)$.

The distances from the PW to the OF were classified into groups that were $0.5 \mathrm{~cm}$ apart. The increasing distances of the alopecia from the center of the PW were accumulated and analyzed by using a table and a graph (Table 6, Fig. 2).

Among the 322 subjects, there were 2 patients who had a distance of $\geq 5 \mathrm{~cm}(5.1 \mathrm{~cm}$ and $5.5 \mathrm{~cm})$, but no one had a distance of $\geq 5.5 \mathrm{~cm} .99 .4 \%$ of 322 subjects had a distance of less than $5 \mathrm{~cm}$. Subjects with a distance of $4 \mathrm{~cm}$ showed an accumulation rate of $93.8 \%$, those with $4.3 \mathrm{~cm}$ showed a rate of $95 \%$, those with $4.5 \mathrm{~cm}$ showed a rate of $97.5 \%$, and those with $5 \mathrm{~cm}$ showed $99.4 \%$.

The two cases that showed a distance of $\geq 5 \mathrm{~cm}$ were subjects who were 74 years old and 75 years old, and they had had alopecia of Norwood type 6 for 35 years and 40 years, respectively.

It should be noted that among the elderly age group and the group having many cases of alopecia $\geq 20$ years, $99.4 \%$ had a PW-OF distance of less than $5 \mathrm{~cm}$.

As mentioned above, the position of the PW showed great variation among individuals.

\section{Fig. 2. Frequency of distances from $P W$ to $O F$}

The distances from the PW to the OF were classified into groups in increments of $0.5 \mathrm{~cm}$. PW, parietal whorl; $\mathrm{OF}$, occipital fringe.

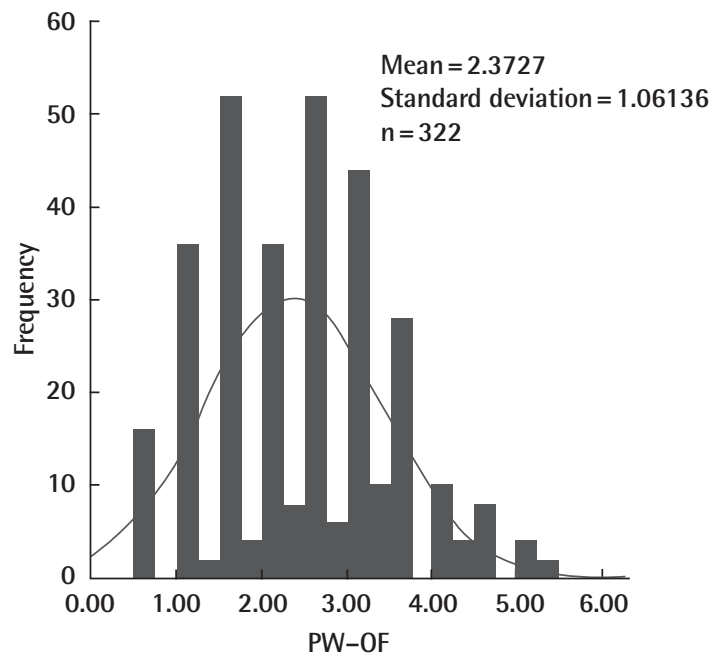

The results of this study are as follows: 1) The position of the PW varied considerably depending on each individual. The center of the PW was positioned at an average distance of 6.25 $\mathrm{cm}$ from the vertical bimeatal line, ranging from a minimum of $1.5 \mathrm{~cm}$ to a maximum of $11 \mathrm{~cm}$. 2) In terms of alopecia progression, no subject showed a distance from the PW to the OF that was greater than $5.5 \mathrm{~cm}$ in this study. Only two patients among 322 subjects showed distances greater than $5 \mathrm{~cm}$, at $5.1 \mathrm{~cm}$ and $5.5 \mathrm{~cm}$. Distances of less than $5 \mathrm{~cm}$ accounted for $99.4 \%$ of all 322 subjects. Cumulative rates were $93.8 \%$ for $4 \mathrm{~cm}, 95 \%$ for 4.3 $\mathrm{cm}, 97.5 \%$ for $4.5 \mathrm{~cm}$, and $99.4 \%$ for $5 \mathrm{~cm}$. No patient surpassed a distance greater than $6 \mathrm{~cm}$ from the PW to the OF.

\section{DISCUSSION}

Hair transplantation has been a long-standing treatment of MPB. Okuda [2] reportedly performed the first surgical hair transplantation in 1939 in Japan. Subsequently, Orentreich [3] was the first American physician to perform hair transplantation for MPB. He suggested a "donor dominance" theory in his 
paper, which was published in 1959. This theory stated that the transplanted hair keeps the original nature of the donor site even after being transplanted. This donor dominance theory became the most fundamental theoretical base of modern hair transplantation. In 1975, Norwood [4] presented the classification and incidence of MPB.

The theoretical foundation that made modern hair transplantation possible, besides the donor dominance theory of Orentreich, is the definition of a safe donor area. No one has ever been able to exactly define the safe donor area, that is, the area that will have no hair loss. No safe donor area guarantees that the hair will be permanent. There exist only safe donor areas anticipated to have no invasion of alopecia. Thus, the definition of the safe donor area, an area in in which no progression of permanent hair loss occurs, is the underlying basis for the practice of hair transplantation surgery [5].

The donor area has three significant boundaries.

First, the anterior boundary of the donor site is usually thought of as vertically superior to that of the external acoustic meatus. Second, the superior boundary of the donor area is located $2 \mathrm{~cm}$ above the upper border of the HR of the horizontal plane. Third, the inferior border of the donor area is slightly controversial, since the inferior margin may move upward with the passage of time [6].

The most crucial and clinically critical standard for determining a safe donor area is the superior border. The superior boundary is profoundly related to the maximum extent of vertex alopecia. The superior margin depends on the degree of vertex alopecia to the occipital side. However, the superior border is comprehensively and indirectly predictable by integrating various factors, including age, family history, physical examination, and finasteride intake.

Some patients exhibit no progression of alopecia in the stages of Norwood types IV and V for more than 20 to 30 years. In contrast, invasion of alopecia has been occasionally observed even in patients in their 20s, exceeding the superior border of the safe donor area, an area that is considered safe.

Numerous hair transplant surgeons have mostly focused on finding reliable factors that enable the prediction of the maximum area of hair loss.

The theory presented by Unger [1] in 1994 is an adaptation of the definition of the safe donor area, which is currently being applied globally to surgical practice by most physicians.

Unger defined alopecia as a continuous progressive condition and proposed the most reasonable standard by calculating the probability of the worst-case scenario.

Unger suggested in accordance with the facts that the global mean life expectancy was $\leq 80$ years old and $>80 \%$ of men aged between 70 to 79 years manifested baldness that is less than Norwood type VII [1]. Unger's theory is still acknowledged as an established theory and hair transplantation surgery in MPB is being implemented on the basis of this definition of the safety zone. Moreover, Bernstein and Rassman [7] also reported on the donor area in hair transplantation surgery and suggested that the apparent density remained the same, although approximately $50 \%$ of the hair was harvested from the permanent safe donor area. They also reported that the safe donor area accounted for approximately $25 \%$ of the entire scalp, and up to $50 \%$ of the hair could be obtained from the donor site $[6,7]$.

Jimenez and Ruifernandez [8] reported that the mean density of the occipital donor area was 65 to $85 \mathrm{FUs} / \mathrm{cm}^{2}$, on the basis of a mathematical model that could estimate the hair density and the donor size of the follicular unit existing in the occipital donor area.

Furthermore, Cole suggested a definition of the donor area during an FUE procedure. According to Cole, the total permanent donor area was $203 \mathrm{~cm}^{2}$. Further, he reported the hair counts existing in the donor area in a Caucasian population by dividing the donor area into eight major regions $(3.5 \mathrm{~cm} \times 6$ $\mathrm{cm})$ and 6 minor regions $(3.5 \mathrm{~cm} \times 2 \mathrm{~cm})$.

Pediatricians and behaviorists have mainly investigated the relationship between various behavioral traits with the position (right or left) of the PW, the orientation of rotation, the incidence of a double whorl, and other factors. Klar et al. [912] published a number of research papers on the presence or the absence of relevance between dominant handedness and PW. One of their studies reported that PW was associated with hemispheric language dominance and homosexuality $[13,14]$. Further, numerous studies investigated PW. However, all of these studies primarily focused on examining or classifying the number of PWs, their positions (right or left), shapes, and/or patterns [15]. However, studies on the relationship between $\mathrm{PW}$ and MPB have not been performed at all.

According to embryology, human hair is known to develop from two ocular punctae and from PW, and directional patterning is determined as permanent hair is fixed in place between the 10th and the 18 th weeks of pregnancy $[16,17]$.

In this period, the PW is the center point for the hair development of the vertex area from where the skull and the brain grow. The scalp skin shows a dome-like growth centering on the whorl, and as the dome-like growth of the scalp skin is faster than the descending speed of the hair follicle roots, the angle and directionality of hair recline to a typical angle $[17,18]$.

Therefore, PW becomes an important anatomical, embryological, or physiological point for determining the development and characteristics of hair on the vertex, in terms of embryology. 
Since the role and physiologic mechanism of the PW in MPB remains unclear, additional studies are thought to be necessary. Noticeably, all vertex types of alopecia initiate from the center of the PW and gradually expand in a radial form. Except for rare cases, such as alopecia totalis and alopecia areata, vertex alopecia of MPB starts from the center of the PW and spreads out in

\section{Fig. 3. A 44-year-old male with typical vertex alopecia}

All vertex types of alopecia initiate from the center of the parietal whorl and gradually expand outward radially.

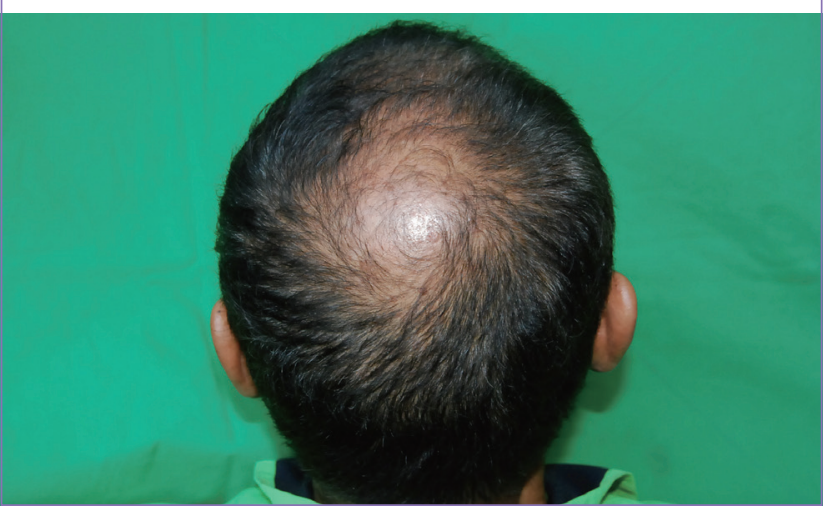

a radial form (Fig. 3).

Donor supply is the ultimate limiting factor in hair transplantation. Every possible effort needs to be made to meet the maximum donor yield.

The available donor area $(\mathrm{C})$ is the area excluding the maximum area of alopecia (B) from the total hair-bearing scalp area (A) of patients with MPB.

$\mathrm{A}-\mathrm{B}=\mathrm{C}$

Since area (B) is unknown, the safe donor area $\left(C^{\prime}\right)$ is the anticipated maximum area of $(C)$ obtained by forecasting the maximum extent of alopecia progression (B). The safe donor area decreases as alopecia spreads out to the occipital area. In contrast, the safe donor area increases as the alopecia progression to the occipital area decreases. The progression range of vertex alopecia to the occipital side is the most critical factor in establishing the safe donor area. The objective and scientific prediction of the progression of vertex alopecia to the occipital side will allow the anticipation of the maximum progression range of vertex alopecia and the available safe donor area of each patient.

According to this study, the position of the PW could provide useful information about the maximum area of (B).

Fig. 4. Case of anteriorly positioned PW

$(A, B)$ A 45-year-old male with male pattern baldness since his early 20 s. (C) He has a very anteriorly positioned PW with a VM-PW distance of $3 \mathrm{~cm}$ and a PW-HR distance of $10.5 \mathrm{~cm}$. VM, vertical bimeatal line; PW, parietal whorl; HR, upper border of helical rim; OF, occipital fringe; SDA, Safe Donor area.
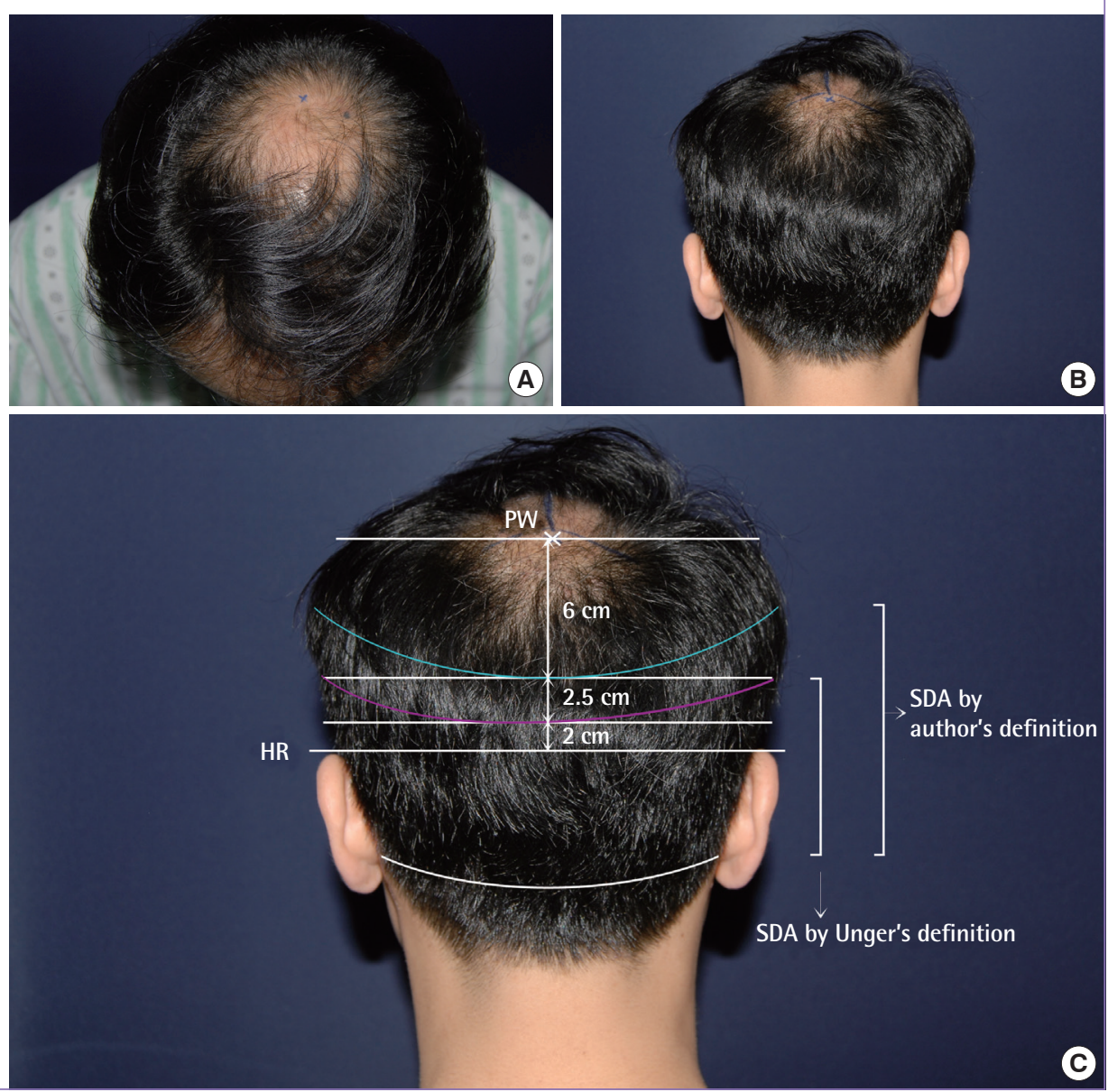
When the PW is anteriorly positioned, vertex alopecia initiates more anteriorly, and when the PW is positioned closer to the occipital side, vertex alopecia progresses posteriorly. Thus, the progression range of alopecia is verified to occur more extensively when the PW is positioned closer to the occipital side. Even though the PW may be anteriorly positioned, if the alopecia of the vertex area progressively spreads to the occipital side, the outcome becomes the same irrespective of the PW position. Therefore, the range of the alopetic area from the PW to the occipital side is crucial. Among the 1,008 Korean male adults considered in the study, no subject had a distance of more than $6 \mathrm{~cm}$. More broad studies involving Caucasians and other races are believed to be essential.

We examined the clinical significance of the study results.

For example, suppose that there is a 23-year-old male with a VM-PW distance of $2.5 \mathrm{~cm}$, a PW-HR distance of $10.5 \mathrm{~cm}$, and Norwood stage III MPB (Fig. 4). This sort of combination happens very frequently. Although it varies depending on the surgeon, the early stage of the manifestations and the young age of the subject are not suitable indications for hair transplantation.
The authors considered that if the maximum progression range of alopecia was less than $5.5 \mathrm{~cm}$, the safest donor area was $6 \mathrm{~cm}$ from the PW. If the maximum distance is set at $5.5 \mathrm{~cm}$, the available safe donor area is assumed to be $5 \mathrm{~cm}$ from the upper border of the HR to the superior border, which is the area that remains after excluding $5.5 \mathrm{~cm}$ from the total of $10.5 \mathrm{~cm}$, which is the PW-HR distance. Thus, the width of the safe donor area increases by $3 \mathrm{~cm}$ from the conventional safe donor area. Suppose that the length of the total donor area is $28 \mathrm{~cm}$, the available donor area is $84 \mathrm{~cm}^{2}\left(3 \times 28 \mathrm{~cm}=84 \mathrm{~cm}^{2}\right)$. When 75 grafts exist per square centimeter, $6,300(75 \times 84=6,300)$ more grafts are available in the donor site, indicating that 3,150 grafts are obtainable when half of the grafts can be harvested. Theoretically, 3,150 grafts can be transplanted in $63 \mathrm{~cm}^{2}$ at a density of 50 grafts per square centimeter, and in $126 \mathrm{~cm}^{2}$ at a density of 25 grafts per square centimeter.

Of course, the fact should be noted that the total recipient area decreases as the PW is more anteriorly positioned.

We can consider the opposite case.

For example, suppose that there is a 23-year-old male with a

\section{Fig. 5. A case of a posteriorly positioned PW}

(A-C) A 31-year-old male with a very posteriorly positioned PW with a VMPW distance of $11.5 \mathrm{~cm}$ and $P W$ $H R$ distance of $2 \mathrm{~cm}$. VM, vertical bimeatal line; PW, parietal whorl; $H R$, upper border of helical rim; OF, occipital fringe; SDA, safe Donor area.
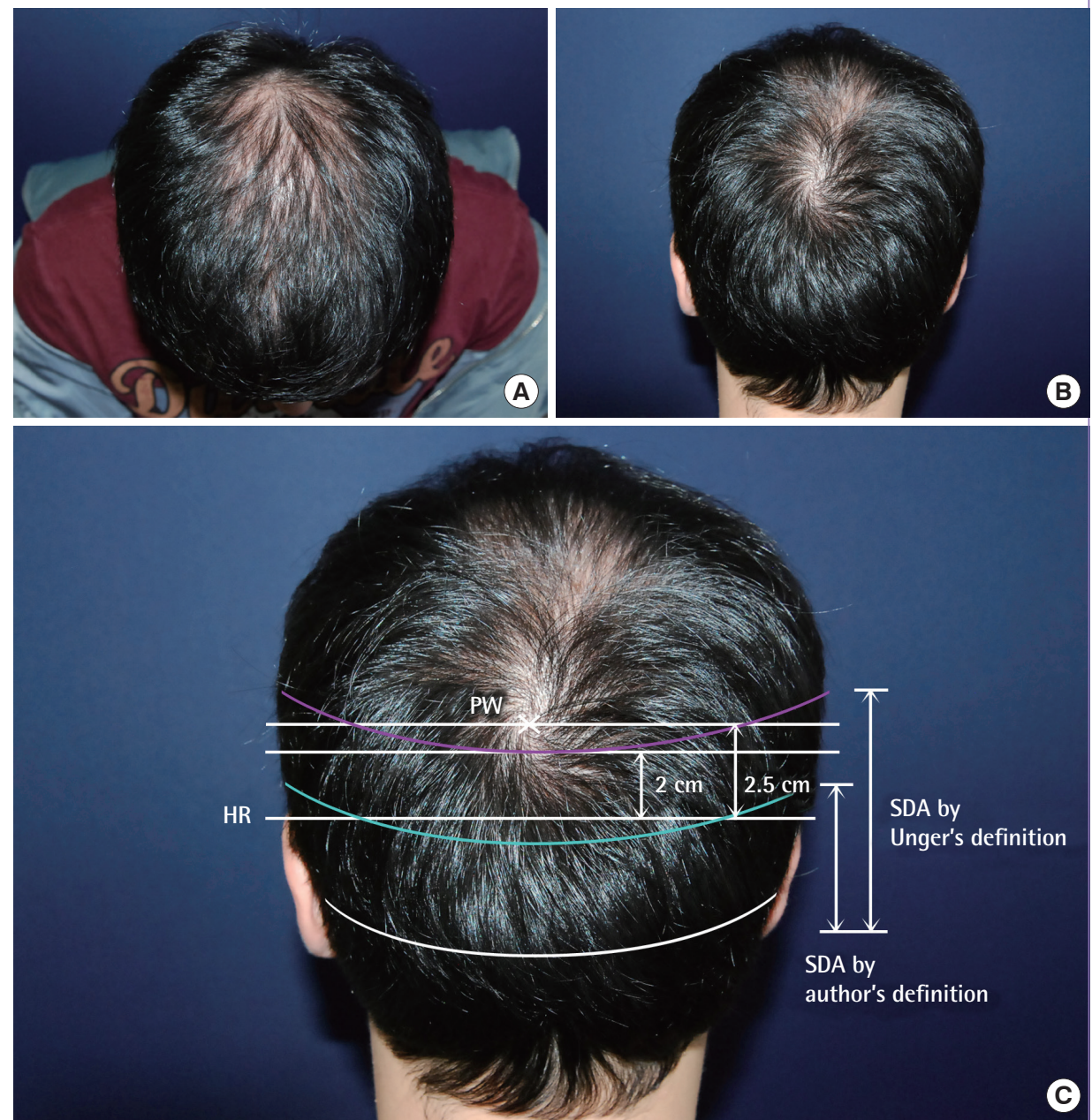
VM-PW distance of $11.5 \mathrm{~cm}$, a PW-HR distance of $2.5 \mathrm{~cm}$, Norwood stage IV MPB, and a father who is Norwood stage VII (Fig. 5).

The patient does not meet the criteria for hair transplantation because of the increased total recipient area and the decreased available donor area.

Likewise, the position of the PW provides very useful information to hair surgeons.

The basic indications for the selection of patients for hair transplantation and the operative planning of the surgical procedure will be altered in many aspects since the total alopetic area and the safe donor area are considerably influenced by the position of the PW.

The starting point, the extent of progression, and the aspects of alopecia in the parietal area can change according to the position of the PW.

According to this study involving 1,008 Korean adult males, no subject exhibited a distance from the PW to the occipital side of more than $6 \mathrm{~cm}$ even when the PW was more anteriorly positioned.

First, alopecia starts from the center of the PW, and then, the alopetic area gradually spreads out in a radial pattern. Second, the location of the PW varies considerably depending on the individual. Third, alopecia mostly progresses within $5.5-6 \mathrm{~cm}$ of the PW toward the occipital side.

If the above factors are scientifically correct, the progression range of the alopecia can be predicted in an objective and scientific manner.

Further studies are considered crucial. However, the finding that there is variation of the maximum progression range of alopecia depending on the position of the PW is of significance to the authors.

It is important to emphasize that the total safe donor area still remains unclear for all patients.

We think that the maximum area of MPB progression and the safe donor area vary according to the position of the PW. Largescale population studies are thought to be essential for further investigations of the PW. Further, Caucasians have a dolichocephalic skull type, with a narrow and vertically long face and skull, unlike the brachycephalic type of Koreans. Therefore, more studies are required for assessing whether the same findings reported from the studies of Caucasians are applicable to other race and ethnic groups.

\section{REFERENCES}

1. Unger W. Delineating the safe donor area for hair transplanting. J Am Acad Cosmet Surg 1994;4:239-43.
2. Okuda S. Clinical and experimental studies of transplantation of living hairs. Jpn J Dermatol Urol 1939;46:135-8.

3. Orentreich N. Autografts in alopecias and other selected dermatological conditions. Ann N Y Acad Sci 1959;83:46379.

4. Norwood OT. Male pattern baldness: classification and incidence. South Med J 1975;68:1359-65.

5. Knudsen RG. The donor area. Facial Plast Surg Clin North Am 2004; 12:233-40.

6. Rassman WR, Carson S. Micrografting in extensive quantities. The ideal hair restoration procedure. Dermatol Surg 1995;21:306-11.

7. Bernstein RM, Rassman WR. Follicular transplantation: patient evaluation and surgical planning. Dermatol Surg 1997;23:771-84.

8. Jimenez F, Ruifernandez JM. Distribution of human hair in follicular units. A mathematical model for estimating the donor size in follicular unit transplantation. Dermatol Surg 1999;25:294-8.

9. Klar AJ. Human handedness and scalp hair-whorl direction develop from a common genetic mechanism. Genetics 2003; 165:269-76.

10. Klar AJ. A single locus, RGHT, specifies preference for hand utilization in humans. Cold Spring Harb Symp Quant Biol 1996;61:59-65.

11. Klar AJ. A 1927 study supports a current genetic model for inheritance of human scalp hair-whorl orientation and hand-use preference traits. Genetics 2005;170:2027-30.

12. Klar AJ. Excess of counterclockwise scalp hair-whorl rotation in homosexual men. J Genet 2004;83:251-5.

13. Devroyre J. An overview of donor area: basic principles. In: Unger W, Shapiro R, Unger R, et al., editors. Hair transplantation. New York: Informa Healthcare; 2011. p.258-9.

14. Perelle IB, Ehrman L, Chanza M. Human handedness and scalp hair whorl direction: no evidence for a common cause. Laterality 2009; 14:95-101.

15. Schmidt H, Depner M, Kabesch M. Medial position and counterclockwise rotation of the parietal scalp hair-whorl as a possible indicator for non-right-handedness. ScientificWorldJournal 2008;8:848-54.

16. Ziering C, Krenitsky G. The Ziering whorl classification of scalp hair. Dermatol Surg 2003;29:817-21.

17. Smith DW, Gong BT. Scalp-hair patterning: its origin and significance relative to early brain and upper facial development. Teratology 1974;9:17-34.

18. Barth JH. Normal hair growth in children. Pediatr Dermatol $1987 ; 4: 173-84$ 